\title{
Gene-editing method tackles HIV in first clinical test
}

\section{Enzymes alter immune cells with no apparent side effects.}

\section{Sara Reardon}

05 March 2014

\author{
NIBSC/Science Photo Library \\ HIV attacks a type of immune cell known as a T cell (shown here) using a protein encoded by the CCR5 \\ gene.
}

A clinical trial has shown that a gene-editing technique can be safe and effective in humans. For the first time, researchers used enzymes called zinc-finger nucleases (ZFNs) to target and destroy a gene in the immune cells of 12 people with HIV, increasing their resistance to the virus. The findings are published today in The New England Journal of Medicine ${ }^{1}$.

"This is the first major advance in HIV gene therapy since it was demonstrated that the 'Berlin patient' Timothy Brown was free of HIV," says John Rossi, a molecular biologist at the Beckman Research Institute of the City of Hope National Medical Center in Duarte, California. In 2008, researchers reported that Brown gained the ability to control his HIV infection after they treated him with donor bone-marrow stem cells that carried a mutation in a gene called CCR5. Most HIV strains use a protein encoded by CCR5 as a gateway into the T cells of a host's immune system. People who carry a mutated version of the gene, including Brown's donor, are resistant to HIV.

But similar treatment is not feasible for most people with HIV: it is invasive, and the body is likely to attack the donor cells. So a team led by Carl June and Pablo Tebas, immunologists at the University of Pennsylvania in Philadelphia, sought to create the beneficial CCR5 mutation in a person's own cells, using targeted gene editing.

\section{Personalized medicine}

The researchers drew blood from 12 people with HIV who had been taking antiretroviral drugs to keep the virus in check. After culturing blood cells from each participant, the team used a commercially available ZFN to target the CCR5 gene in those cells. The treatment succeeded in disrupting the gene in about $25 \%$ of each participant's cultured cells; the researchers then transfused all of the cultured cells into the participants. After treatment, all had elevated levels of T cells in their blood, suggesting that the virus was less capable of destroying them.

Six of the 12 participants then stopped their antiretroviral drug therapy, while the team monitored their levels of virus and T cells. Their HIV levels rebounded more slowly than normal, and their T-cell levels remained high for weeks. In short, the presence of HIV seemed to drive the modified immune cells, which lacked a functional CCR5 gene, to proliferate in the body. Researchers suspect that the virus was unable to infect and destroy the altered cells.

"They used HIV to help in its own demise," says Paula Cannon, who studies gene therapy at the University of Southern California in Los Angeles. "They throw the cells back at it and say, "Ha, now what?"

\section{Long-term action}

In this first small trial, the gene-editing approach seemed to be safe: Tebas says that the worst side effect was that the chemical used in the process made the patients' bodies smell bad for several days.

The trial "isn't the end game, but it's an important advance in the direction of this kind of research", says Anthony Fauci, director of the US National Institute of Allergy and Infectious Diseases in Bethesda, Maryland. "It's more practical and applicable than doing a stemcell transplant," he says, although it remains to be seen whether it is as effective.

Tebas says that the team's ultimate goal is to wean some people off antiretroviral drugs all together. He and June are particularly excited about one study participant in whom the virus did not return during the 12-week portion of the study in which antiretroviral therapy was paused. When they examined his genome, they found that he already had one mutated copy of CCR5. "Nature had done half of the job," says Tebas. After the addition of the modified cells, well over half the T cells in his body were resistant to HIV. 
The finding suggests that people with only one functional copy of CCR5 might be particularly good candidates for this therapy, and the researchers have now begun enrolling people in such a trial. June says that the team is also trying to find ways to increase the number of cells that the ZFNs modify, and to help the cells to proliferate.

June expects the field of gene therapy to grow: newer enzymes on the market, such as transcription activator-like effector nucleases (TALENs) and clustered regularly interspaced palindromic repeats (CRISPRs), can target genes more precisely than ZFNs, and can replace DNA rather than simply disrupting genes. He concedes that widespread clinical use of such enzymes is several years off, but expects researchers to start to look at their potential for altering cells in people with disorders that result from mutations in a single gene, such as sickle cell anaemia, certain types of cancer and even metabolic diseases in the brain.

"I get goosebumps thinking about the potential of this technology," says Cannon. Her group has been using ZFNs to target CCR5 in haematopoietic stem cells, which can turn into several types of immune cell and might allow patients to make their own unending supply of resistant cells. Cannon and her colleagues hope to start a trial of these cells later this year.

Nature | doi:10.1038/nature.2014.14813

\section{References}

1. Tebas, P. et al. New Engl. J. Med. 370, 901-910 (2014). 